OAR $93 / 4$

gr-qc/9303023

\title{
Inflationary attractors and perturbation spectra in generally coupled gravity
}

\author{
Luca Amendola, Diego Bellisai and Franco Occhionero \\ Osservatorio Astronomico di Roma \\ Viale del Parco Mellini, 84 \\ I-00136 Rome, Italy
}

\begin{abstract}
A generic outcome of theories with scalar-tensor coupling is the existence of inflationary attractors, either power-law or de Sitter. The fluctuations arising during this phase are Gaussian and their spectrum depends on the wavenumber $k$ according to the power-law $k^{1 /(1-p)}$, where $p$ is the inflationary power-law exponent. We investigate to which extent these properties depend on the coupling function and on the potential. We find the class of models in which viable attractors exist. Within this class, we find that the cosmic expansion and the scaling of the fluctuation spectrum are independent of the coupling function. Further, the analytical solution of the Fokker-Planck equation shows that the deviations from Gaussianity are negligible.
\end{abstract}

Accepted Feb. 24, 1993

To be published on Physical Review D.

PACS: 98.80.Bp, 98.80.Dr 


\section{Introduction}

Non-minimally coupled theories of gravity are models in which a direct coupling of the form

$$
A_{N M C}=\int d^{4} x \sqrt{-g}\left[\frac{1}{2} \xi f(\phi) R\right]
$$

between a matter field $\phi$ and the curvature scalar $R$ is added to the gravity Action. The interesting history of such theories in general relativity and cosmology dates back to the Dirac suggestion and to Jordan-Brans-Dicke (JBD) modeld1. Terms with non-minimal coupling (NMC) arise quantizing fields in curved space2, in multi-dimensional theories like superstrings or supersimmetry and in induced gravity theories 4 . On the other hand, La and Steinhardt's extended inflation 5 (EI) and many variants within the context of 'first-order inflation' 6 reconcile a first-order phase transition with inflation modulating the bubble nucleation rate. The cosmological kinematics of NMC theories is also discussed in Ref. (7).

No known fundamental principle predicts the functional form of $f(\phi)$. One can find in literature many ansätze: $f(\phi)=\phi^{2}$, as in the induced gravity model and some version of EI; $f(\phi)=a+b \phi^{2}+c \phi^{4}+\ldots$, as in the hyperextended design of EI; $f(\phi)=e^{D \phi}$, as in dimensionally reduced Kaluza-Klein theories, and so on. In Ref. [9], non-minimal derivative couplings in $f(\phi)$ have also been taken into account. Models with a generalized kinetic term like $\omega(\phi) \phi_{; \mu} \phi^{; \mu} / \phi$ and a coupling term $\Phi R$ have also been considered (the case $\omega=$ const is indeed the JBD theory and the original formulation of EI); up to a redefinition of $\phi$ these models are equivalent to introducing a coupling like (11). Aim of this paper is to investigate in a systematic fashion the dynamical features of models with a general coupling $f(\phi)$, without confining ourselves to a specific choice, except for the following requests: that in the large $\phi$ limit the function $f(\phi)$ grows faster than $\phi^{2}$ (the case $f(\phi) \sim \phi^{2}$ being already well-known), and that the potential $V(\phi)$, at least for large $\phi$, can be written as

$$
V(\phi)=\lambda f^{M}(\phi)
$$

where $\lambda$ and $M$ are arbitrary non-negative constants. 0 We also assume $V(\phi), f(\phi) \geq 0$, the equality being verified when $\phi$ eventually falls into its stable minimum. It is remarkable that, with the above assumptions, it is possible to find analytically, without specifying nor the coupling nor the potential, the class of inflationary attractors, the order-of-magnitude amplitude of the primordial perturbations and the solution of the Fokker-Planck equation for the stochastic fluctuations. Since $f(\phi)$ is left largely unspecified, we speak here of theories with general coupling, instead of non-minimal coupling models. We also give examples of numerical phase-space (PS) of the investigated models.

The plausibility of inflation depends on how large is the set of initial conditions which evolve to inflationary expansion of the scale factor: the existence of an inflationary attractor extending to values of the field as large as possible, up to the Planck boundary, is then a very desirable property of a cosmological model. A first result of the present paper is that we determine the class of models with coupling different from the standard choice $\phi^{2} R$ which have asymptotic inflationary attractors. We find that, in most models, either the Universe does not inflate at all, or it does so toward the wrong phase-space direction. The main

${ }^{a}$ After this work was completed we learned of independent work of De Ritis and coworker 10 , who obtained exact solutions in particular cases of the above functional relation. 
result is that, in those cases in which a successful inflation is recovered, the kinematics of the Universe and the main features of the fluctuation spectrum do not depend on $f(\phi)$.

\section{Inflationary attractors and the perturbation spectrum}

We start from the following Action [we assume Planck units, $G=c=\hbar=1$ and signature $(+---)]$

$$
A=\int d^{4} x \sqrt{-g}\left[-\frac{R}{16 \pi}+\frac{1}{2} \xi f(\phi) R+\frac{1}{2} g^{\mu \nu} \phi_{; \mu} \phi_{; \nu}-V(\phi)\right] .
$$

Great simplification is obtained passing to the the so-called Einstein frame, i.e. deriving the field equation in the new metric $\tilde{g}_{\mu \nu}=e^{2 \omega} g_{\mu \nu}$ with

$$
2 \omega=\ln |1-\gamma f|
$$

where $\gamma \equiv 8 \pi \xi<0$. We will work here assuming $\xi<0$, which guarantees that the new metric is non-singular. The old metric will be referred to as the Jordan frame. The Einstein equations in the new frame are then

$$
\tilde{G}_{\mu \nu}=\frac{1}{1-\gamma f}\left[8 \pi T_{\mu \nu}+\frac{3 \gamma^{2} f^{\prime 2}}{2(1-\gamma f)}\left(\phi_{; \mu} \phi_{; \nu}-\frac{1}{2} g_{\mu \nu} \phi_{; \alpha} \phi^{; \alpha}\right)\right],
$$

where $T_{\mu \nu}$ is the usual energy-momentum tensor for the scalar field $\phi$. From now on, we confine ourselves to a homogeneous, isotropic and spatially flat metric with cosmic factor $a(t)$ and Hubble function $H(t)=\dot{a} / a$. Unless otherwise stated, all quantities are meant to be expressed in the rescaled variables: a dot denotes differentiation with respect to the new time $t=\int e^{\omega\left(t^{\prime}\right)} d t^{\prime}$, the Hubble function $H$ is in terms of the new cosmic factor, and so on. If $K_{\mu \nu}=\phi_{; \mu} \phi_{; \nu}-\frac{1}{2} g_{\mu \nu} \phi_{; \alpha} \phi^{; \alpha}$ denotes the kinetic sector of $T_{\mu \nu}$ in the original frame, the kinetic sector $\tilde{K}_{\mu \nu}$ in the new Einstein frame can be written in the form $\tilde{K}_{\mu \nu}=F^{2}(\phi) K_{\mu \nu}$, where

$$
F^{2}(\phi)=\left[16 \pi(1-\gamma f)+3 \gamma^{2} f^{\prime 2}\right] / 16 \pi(1-\gamma f)^{2} .
$$

A canonical kinetic sector is then obtained defining a new field

$$
\psi=\int d \phi F(\phi) .
$$

This integral is not easily done, even with simple choices of the coupling $f(\phi)$. However, a powerful simplification is attained in the limit $|\phi| \rightarrow \infty$, the same limit in which the effect of the coupling is greater and inflationary attractors are found. Let us denote in the following with a subscript ' $i$ ' a quantity evaluated at the initial time. Then, if $f^{\prime 2}$ grows faster than $f$, we can integrate Eq. (7) into $c \psi=\ln \left[f(\phi) / f_{i}\right]$, and thus $f(\phi)=f_{i} \exp c \psi$, where $c=(16 \pi / 3)^{1 / 2}$. It then follows that the initial value of $\psi$ is $\psi_{i}=0$. When $f^{\prime 2}$ grows exactly like $f$, i.e. for $f=\phi^{2}$, one has instead $c(\xi)=2[\gamma /(6 \xi-1)]^{1 / 2}$.

Suppose now that the following relation holds between the coupling $f$ and the potential: $V=\lambda f^{M}$. This relation is verified, for instance, if both $V$ and $f$ are power functions of the 
field, or if they are both exponentials. The potential in the Einstein frame, in the same limit as above, is then

$$
U(\psi)=\lambda \gamma^{-2} f^{\mu}=\beta \exp (c \mu \psi),
$$

where $\beta \equiv f_{i}^{\mu} \lambda \gamma^{-2}$, and $\mu \equiv M-2$. We then explore the bidimensional parameter space $(\xi, M)$. Remarkably, an asymptotic flat potential able to drive a slow-rolling inflation in a FRW metric with scale factor $a(t)$ is found in a single case: $V \sim f^{2}$. In all other cases, we find in the $\dot{\psi}, \psi$ phase space the attractor trajectories

$$
\dot{\psi}=A e^{c \mu \psi / 2}, \quad A^{2}=2 \beta \mu^{2} /\left(9-\mu^{2}\right),
$$

where the negative root for $A$ is to be chosen, corresponding to $\phi$ decreasing toward its stable minimum, where the Friedmann behavior takes place. This corresponds to trajectories

$$
f^{\prime} \dot{\phi}=c A f_{i}^{-\mu / 2} f^{(\mu+1) / 2}
$$

in the $(\dot{\phi}, \phi)$ plane. In the Appendix, solutions of this kind will be proven to be attractors; graphic evidence of their attractive properties is provided by our Poincarè projected phase spaces (Figs. 1-4). A power-law expansion (for $M \neq 2$ ) takes place on the attractors:

$$
a=a_{i}(1+t / \tau)^{p}, \quad p=3 / \mu^{2}
$$

(in the conformally rescaled time), where $\tau=-2 / A c \mu>0$. The cancellation of the coupling constant $\xi$ from (11) occurs only in this class of models: in the ordinary coupling $f=\phi^{2}$ the cancellation does not take place. Notice that the solution (111) as well as the attractor (9) above are exact solutions as long as we take the potential (8). From (11) we see that $H=H_{i}(1+t / \tau)^{-1}$, where $H_{i}=p / \tau$.

The behavior of the cosmic scale factor is accelerated, hence inflationary, only if $M<$ $\sqrt{3}+2 \sim 3.7$. However, for $M<2$ the model enters an eternally inflating phase, since then $d U / d \psi<0$ and the field $\psi$ is pushed to ever growing values, never reaching the Friedmann phase located at $\psi \rightarrow-\infty$ [from the mapping $\psi \rightarrow \phi$ follows that $\psi \rightarrow-\infty$ when $f(\phi)$ vanishes]. The only allowed range is then

$$
2 \leq M<3.7 \text {. }
$$

Outside this narrow range, the model studied here does not allow a successful chaotic inflation. When $f=\phi^{2}$ the expansion is modified to $\hat{p}=p(1-1 / 2 \xi)$. This is inflationary for any $M$ if $\xi<0$ is small enough 5 .

In the original Jordan frame the cosmic scale expansion is still a power-law in the Jordan time, but with an exponent $p_{J}=(p+\mu) /(1+\mu)$, which is inflationary if $p>1$.

Since we have our theory in the Einsteinian form, we are allowed to employ the standard formalism 19 to derive the perturbation spectrum at the horizon-crossing epoch. We will see that we can determine the spectrum without specifying the coupling function. We assume here that the horizon-crossing condition is to be found in the Einstein frame, i.e. that the condition only involves the rescaled variables [see Ref. (11)].

The rms amplitude $\delta \rho / \rho$ of the perturbations on a comoving scale $k^{-1}$ at horizon crossing (after the end of inflation) is given by

$$
\delta \rho / \rho=\left(H^{2} / \dot{\psi}\right)_{k=a H},
$$


times an order-of-unity factor 1 , 目 depending on the power-law exponent $p$. Along the solution (9) we have

$$
H^{2} / \dot{\psi}=(\tau / 2) c \mu H_{i}^{2}(1+t / \tau)^{-1}
$$

Now, since $k=a H=a_{i} H_{i}(1+t / \tau)^{p-1}$, eliminating the time between the latter equation and (14) we obtain

$$
\delta \rho / \rho=(p / 2) c \mu H_{i}\left(k / a_{i} H_{i}\right)^{1 /(1-p)},
$$

where the last factor expresses the scale dependence of the spectrum. Since $p>1$, the spectrum grows with the scale, as it is commonly found in power-law inflation, and becomes flat for $p \rightarrow \infty$ (slow-rolling inflation). Our result shows that this conclusion does not depend on the choice of $f(\phi)$, as long as $V=\lambda f^{M}$.

\section{$3 \quad$ Stochastic approach to fluctuations}

To compare the theory with the observed large-scale structure we need also the higher-order moments of the fluctuation distribution. In particular, it is astronomically interesting to detect possible deviations from Gaussianity of the primordial spectrum 16 . We investigate then the stochastic properties of adiabatic fluctuations during a power-law inflation driven by the JBD field $\phi$. The pioneering work in this context is Ref. (17). Since we have now an Einsteinian theory with an exponential potential14, we may analytically solve the Fokker-Planck equation (FPE) for the probability distribution of the fluctuations. The only other known cases of analytically solvable FPE are the minimally coupled $[f(\phi)=0]$ case with quartic or exponential potentials 15 . In power-law inflation, contrary to slow-rolling inflation, the term $\ddot{\psi}$ is not generally negligible in the field equation for $\psi$. However, we show that its contribution is not dominant in the cases investigated here, at least for $M$ not close to its upper bound 3.7. The condition $|\ddot{\psi}| \ll\left|U^{\prime}\right|$ gives in fact, when applied to the attractor solution (9), $A^{2} / 2 \beta \ll 1$, that is, $(c \mu)^{2} \ll 24 \pi$. We have, for instance, $A^{2} / 2 \beta \sim 0.12$ for $M=3$, which is moderately satisfactory, and $A^{2} / 2 \beta \sim 0.03$ for $M=2.5$, a good approximation. We can then safely put $\dot{\psi}=-U^{\prime} / 3 H$ and write the Langevin equation in the useful time variable $\alpha=\int H d \tau$

$$
\frac{d \psi}{d \alpha}=-\frac{U^{\prime}}{3 H^{2}}+\sqrt{\frac{3}{8 \pi}} g H \eta(\alpha)=-\frac{c \mu}{8 \pi}+g \sqrt{\beta} e^{c \mu \psi / 2} \eta(\alpha),
$$

where $\eta(\alpha)$ is the stochastic Gaussian noise due to the super-horizon stretching of high frequency modes, and $g$ is an order-of-unity constant 14 which depends on the power-law exponent $p$. We have made use in (16) of the $(0,0)$ Einstein equation in the Einsteinian frame in slow-rolling regime,

$$
3 H^{2}=8 \pi \lambda \gamma^{-2} f_{i}^{\mu} \exp (c \mu \psi) .
$$

We will denote here with a subscript ' $c l$ ' the unperturbed quantities, i.e. the solution to the zero-noise classical equations of motion, to distinguish them from the stochastic variables 
which appear in the Langevin and Fokker-Planck equations. Let us define the new field variable

$$
\Psi=\int \frac{d \psi}{g \sqrt{\beta} e^{c \mu \psi / 2}}=-\frac{2 \gamma}{c \mu g \sqrt{\lambda}} f_{i}^{-\mu / 2} e^{-c \mu \psi / 2},
$$

from which Eq. (16) for $f \rightarrow \infty$ is simplified to

$$
d \Psi / d \alpha=\Psi / p+\eta(\alpha) .
$$

The FPE associated to Eq. (19) is in the Ornstein-Uhlenbeck 18 form

$$
\frac{\partial W}{\partial \alpha}=-\frac{1}{p} \frac{\partial(\Psi W)}{\partial \Psi}+\frac{\partial^{2} W}{\partial \Psi^{2}}=-\frac{\partial\left(D_{1} W\right)}{\partial \Psi}+\frac{\partial^{2}\left(D_{11} W\right)}{\partial \Psi^{2}},
$$

where $D_{1}=\Psi / p$ is the drift coefficient and $D_{11}=1$ is the diffusion coefficient. One sees that the definition domain of $\Psi$ is $(0,+\infty)$ for $f(\phi)$ non-negative definite (remember that $\gamma<0$ ). Then we must impose the reflecting boundary conditions $S(\Psi=0)=0, S(\Psi \rightarrow \infty)=0$ where

$$
S=-D_{1} W+\partial\left(D_{11} W\right) / \partial \Psi
$$

is the probability flux. This introduces a 'reflecting' term in the solution 14 , which tyrns out to be negligible at large times, when $\langle\Psi\rangle$ moves to infinity. The general solution 18 of (20) with initial condition $W(\Psi, 0)=\delta\left(\Psi-\Psi_{i}\right)$ is then found to be

$$
W(\Psi, \alpha)=\sqrt{\frac{1}{2 \pi p\left(e^{2 \alpha / p}-1\right)}}\left\{\exp \left[-\frac{\left(\Psi-\Psi_{c l}\right)^{2}}{2 p\left(e^{2 \alpha / p}-1\right)}\right]+\exp \left[-\frac{\left(\Psi+\Psi_{c l}\right)^{2}}{2 p\left(e^{2 \alpha / p}-1\right)}\right]\right\}
$$

where $\Psi_{c l}=\Psi_{i} e^{\alpha / p}$. It is easy to see that all the moments of $\psi$ are defined. In fact, the integral

$$
M_{\kappa}(\psi) \equiv<\psi^{\kappa}>=\int_{-\infty}^{\infty} \psi^{\kappa} W(\psi, \alpha) d \psi
$$

where $W(\psi, \alpha)=W(\Psi, \alpha)|J|$, and $|J|=d \Psi / d \psi$, converges for any $\kappa$. The same is not always true for the moments of the original field $\phi$. For instance, if $f(\phi)=\phi^{2 m}$, we have that all the moments $\left\langle\phi^{\kappa}>\right.$ for which $\kappa \geq m \mu$ are divergent for $\phi \rightarrow \infty$.

The distribution of the field $\psi$ is non-Gaussian. We are interested in estimating its deviation from Gaussianity through the skewness coefficient

$$
s=M_{3}\left(\psi-\psi_{c l}\right) / M_{2}^{3 / 2}\left(\psi-\psi_{c l}\right) .
$$

In the following we will neglect the contribution from the reflecting term, so that we have $M_{1}(\psi)=\psi_{c l}$. We may first calculate approximately the second central moment of $\psi$, i.e. the variance, in the limit of small $\left|\psi-\psi_{c l}\right|$. We will see that this is completely justified in viable theories of inflation, due to the smallness of $H$, which sets the amplitude of the diffusion around the classical trajectory. Expanding $\left|\Psi-\Psi_{c l}\right|$ in powers of $\left|\psi-\psi_{c l}\right|$ we can write the solution $W(\psi)$ to the first order as a Gaussian with mean $\psi_{c l}$ and variance

$$
D=p g^{2}\left(e^{2 \alpha / p}-1\right)\left[\lambda \gamma^{-2} f_{i}^{\mu} e^{c \mu \psi_{c l}}\right] .
$$


We can now recognize in the square brackets, apart order-of-unity factors, the classical value of $H^{2}$. It follows that, for large $\alpha$,

$$
\sqrt{<\left(\psi-\psi_{c l}\right)^{2}>} \approx H_{c l} e^{\alpha / p}=H_{c l}(t / \tau) .
$$

For $t \gg \tau$ we have $H_{c l} t / \tau \sim H_{i}$. In order to avoid exceedingly large perturbations on the microwave sky, $H_{i}$ must be smaller than, roughly, $10^{-4}$ in Planck units, as it is evident from Eq. (15). It turns out that the deviation of $\psi$ from $\psi_{c l}$ is indeed very small, which justifies the approximations made above. We may now calculate the third central moment $M_{3}\left(\psi-\psi_{c l}\right)$ in the following way. Let us consider a Gaussian distribution $W(\Psi)$ normalized to unity, with mean $\Psi_{c l}$ and variance $\sigma$. We transform it under the mapping $\Psi=\Psi(\psi)$ to $W[\Psi(\psi)]\left|\Psi^{\prime}\right|$, where $\Psi^{\prime}=d \Psi / d \psi$. The inverse mapping is $\psi=\psi(\Psi)$. The mean of $\psi$ is now $\psi_{c l}=\psi\left(\Psi_{c l}\right)$, while the variance and the higher-order moments have to be calculated. The new distribution is $W(\psi)=W[\Psi(\psi)]\left|\Psi^{\prime}\right|$. In the limit $\left|\psi-\psi_{c l}\right| \rightarrow 0$ we may expand $\Psi(\psi)$ and $\Psi^{\prime}$ in Taylor series around $\psi_{c l}$. It follows

$$
W(\psi)\left|\Psi^{\prime}\right|=(2 \pi D)^{-1 / 2} \exp \left\{-\frac{1}{2 D}\left[\left(\psi-\psi_{c l}\right)^{2}-R\left(\psi-\psi_{c l}\right)\right]\right\},
$$

where $D=\sigma\left|\Psi_{c l}^{\prime}\right|^{-2}$ and $R=2 D \Psi_{c l}^{\prime \prime} / \Psi_{c l}^{\prime}$. Here $\Psi_{c l}^{\prime}, \Psi_{c l}^{\prime \prime}$ mean $\Psi^{\prime}$ and $\Psi^{\prime \prime}$, respectively, calculated in $\psi=\psi_{c l}$. The distribution (27) is again a Gaussian curve, but with mean, variance and normalization different from the original ones. After some simple manipulations it is easy to find

$$
M_{3}\left(\psi-\psi_{c l}\right)=\frac{R}{2}\left(3 D+\frac{R^{2}}{4}\right) .
$$

Let us specialize to our model. We have then that $D$ is given in (25), while $R=-c \mu D$. In the limit of small $D$, i.e. small $H^{2}$, we have

$$
s=-3 c \mu D^{1 / 2} / 2 \sim-H_{c l}(t / \tau) .
$$

The deviation from a Gaussian distribution turns out to be indeed negligible as long as the initial value for $H$ is less than $10^{-4}$ in Planck units. In other words, the need for a smooth microwave background has the consequence of a very small deviation from Gaussianity. Perturbations which crossed out the horizon when $H \sim 1$ can have a very skewed distribution, but they are now much larger than the observable Universe. Similar conclusions hold for the higher moments of the distribution.

\section{Conclusions}

One of the most appealing aspects of chaotic inflation is that even the simplest models possess the required properties for a successful inflation. Almost all the possible initial conditions for a scalar field dominated Universe with a simple potential lead to a conspicuos period of inflation. This feature implies, in the dynamical phase-space of such theories, the existence of attractor trajectories over which the other trajectories converge. It turns out, contrary to the uncoupled case, $f=0$, and to the usual $f=\phi^{2}$ case, that models with generic coupling 
satisfy the chaotic inflation requirements only when $2 \leq M<3.7$. The resulting powerlaw expansion is independent of $f(\phi)$. This power-law case is noticeable because it can be adopted as a chaotic hyperextended inflation with a kinematics independent of the coupling function.

The scale-dependence, $k^{1 /(1-p)}$, also independent of $f(\phi)$, is an interesting signature of power-law inflation, possibly testable with the data from COBE. Solving the Fokker-Planck equation we can evaluate higher-order moments of the fluctuation distribution. This shows that the deviation from Gaussianity on observable scales is negligible in all acceptable inflationary models of the kind studied here.

Finally, we prove analytically in the Appendix the stability of the attractors.

\section{Appendix}

Numerical phase-spaces show with full evidence the stability of the trajectories labelled as $A$-type in the text. Here we prove it analytically for the case $M>2$. As it was shown in Sect. 2, the phase-space in the large $\phi$ limit is governed, in the rescaled variables, by the dynamical system $(x=\psi, y=\dot{\psi})$

$$
\dot{x}=y, \quad \dot{y}=-3 H(x, y) y-U^{\prime}(x),
$$

where $U^{\prime}=\beta c \mu e^{c \mu x}$. There is an attractor trajectory

$$
y_{0}=A e^{c \mu x / 2},
$$

with $c \mu>0$ and $A<0$. Here we assume for simplicity that $H$ is calculated along the attractor $H=\left[8 \pi\left(1 / 2+\beta / A^{2}\right) / 3\right]^{1 / 2}\left|y_{0}\right|$, freezing the $x, y$-dependence of $H$ on the assumed solution (31). Dealing with the full function $H=H(x, y)$ only entangles the algebraic details without changing the conclusions. To show that (31) is indeed an attractor, let us define a new set of coordinates $u, v$, such that $u=0$ defines the trajectory (31): $u=y-A e^{c \mu x / 2}, \quad v=$ $y+A e^{c \mu x / 2}$. Under this mapping, the dynamical system (30) becomes

$$
\begin{aligned}
& 2 \dot{u}=\frac{c \mu}{4}\left(u^{2}-v^{2}\right)-3 H(u+v)-2 U^{\prime}, \\
& 2 \dot{v}=\frac{c \mu}{4}\left(v^{2}-u^{2}\right)-3 H(u+v)-2 U^{\prime} .
\end{aligned}
$$

We now linearize (32) around $x_{0}, y_{0}=A e^{c \mu x_{0} / 2}$, i.e. around a generic point on $A e^{c \mu x / 2}$. This corresponds to linearizing around $u=0, v=v_{0}=2 A e^{c \mu x_{0}}$. Notice that $v_{0}<0$. We get then for the coordinate $u$ the following equation (the equation for $v$ is not of concern in this context)

$$
\dot{u}=a_{1} v+a_{2} u \text {. }
$$

It turns out that $a_{1}=0$ : this confirms that the trajectory $u=0$ is a solution of the system (30). The sign of $a_{2}$ determines the stability of the attractor: when $a_{2}$ is negative, in fact, $u \rightarrow 0$ starting from any small perturbation $u_{0}$. The process evolves with a time-scale $1 /\left|a_{2}\right|$. We have

$$
a_{2}=v_{0} c \mu\left[\left(18-\mu^{2}\right) / 2 \mu^{2}\right]
$$


which is indeed negative because $v_{0}$ is negative and $m<\sqrt{3}$. The stability time scale $1 /\left|a_{2}\right|$ goes like $(\beta)^{-1 / 2}$, i.e. like $\xi \lambda^{-1 / 2}$ : the process of infalling toward the attractor is faster as $\xi \rightarrow 0$ or $\lambda \rightarrow \infty$. 


\section{REFERENCES}

1. P. A. M. Dirac, Proc. R. Soc. A338, 439 (1974) ; P. Jordan, Schwerkraft und Weltall (Wieweg, Braunschweig, 1955); C. Brans and R. H. Dicke Phys. Rev. , 124, 925 (1961).

2. N. D. Birrel and P. C. W. Davies, Quantum Fields in Curved Spaces (Cambridge Univ. Press, Cambridge, 1982).

3. S. Randjbar-Daemi, A. Salam, and J. Strathdee, Phys. Lett. B135, 388 (1984); K. Maeda, Class. Quantum Gravit. 3, 233 (1986); T. Applequist and A. Chodos, Phys. Rev. Lett. 50, 141 (1983).

4. A. Zee, Phys. Rev. Lett. , 42, 417 (1979); F. S. Accetta, D. J. Zoller and M. S. Turner, Phys. Rev. D31, 3046 (1985).

5. D. La and P. J. Steinhardt, Phys. Rev. Lett., 62, 376 (1989).

6. D. La and P. J. Steinhardt, Phys. Lett. B220, 375 (1989); F. S. Accetta and J. J. Trester, Phys. Rev. D39, 2854 (1989); R. Holman, E. W. Kolb, and Y. Wang, Phys. Rev. Lett. 6517 (1990); R. Holman, E. W. Kolb, S. L. Vadas and Y. Wang, CMU-HEP 90-09, FNAL Pub-90/99-A (1990) preprint; A. Linde, CERN-TH-5806/90 preprint (1990); Y. Wang, Phys. Rev. D42, 2541 (1990); L. Amendola, S. Capozziello, M. Litterio and F. Occhionero, Phys. Rev. D45, 417 (1992).

7. U. Kasper, Nuovo Cim. B103, 291 (1989); T. Futamase and K. Maeda,Phys. Rev. D39, 399 (1989); L. Amendola, M. Litterio and F. Occhionero, Int. J. Mod. Phys. A, 5, 3861 (1990).

8. P. J. Steinhardt and F. S. Accetta, Phys. Rev. Lett. 64, 2740 (1990).

9. L. Amendola, preprint (1993), to be published in Phys. Lett. B.

10. R. De Ritis, talk delivered at the "X Italian Conference on General Relativity and Gravitational Physics", Bardonecchia, Sept. 1-5 1992.

11. A. H. Guth and B. Jain, Phys. Rev. D45, 426 (1992); D. H. Lyth and E. D. Stewart, Phys. Lett. B274, 168 (1992).

12. A. Linde, Phys. Lett. 129B, 177 (1983); Nucl. Phys. B216, 421 (1983).

13. B. Whitt, Phys. Lett. 145B, 176 (1984); J. D. Barrow and Cotsakis S.,Phys. Lett., 214B, 515 (1988); K.-I. Maeda, Phys. Rev. D39, 3159 (1989); H.-J. Schmidt, Class. Q. Grav. 7, 1023 (1990).

14. F. Lucchin and S. Matarrese, Phys. Rev. D32, 1316 (1985).

15. A. Ortolan, F. Lucchin and S. Matarrese, Phys. Rev. D38, 465 (1988).

16. W. Saunders et al. , Nature 349,32 (1991).

17. A. A. Starobinsky, in Field Theory, Quantum Gravity and Strings, eds. H.J. de Vega and N. Sanchez (Springer-Verlag, Lect. Notes in Physics 246, 1986)

18. H. Risken, The Fokker-Planck Equation (Springer-Verlag, Berlin, 1989).

19. A. Guth and S.-Y. Pi, Phys. Rev. Lett. 49, 1110 (1982); S. W. Hawking, Phys. Lett. B 115, 339 (1982); A. A. Starobinsky, Phys. Lett. B 117, 175 (1982).

20. G. F. Smoot et al., COBE preprint 92-02 (1992). 


\section{FIGURE CAPTIONS}

1. The phase-space portrait of the model with $f(\phi)=\phi^{2 m}$ and $V(\phi) \sim \phi^{2 n}$, where $n=1, m=2$ and negative $\xi$ is displayed. Notice the two outward directed attractors and the central Friedmann region. This model is outside the successful range of parameters. All the trajectories spring out from the singular points at infinity on the Poincaré circle at angles $\pi / 2$ and $3 \pi / 2$. The diagram is symmetric with respect to the origin.

2. As in Fig. 1, with $n=5, m=2$. This model is inside the successful range: the power-law attractors have a natural end.

3. As in Fig. 1, with $n=4, m=2$. This model satisfies the condition $M=2$. Along the deSitter-like attractors one has $\ddot{\phi}=\dot{\phi} \rightarrow 0$.

4. As in Fig. 1, with $n=0, m=1$. This phase-space is equivalent to extended inflation. 